ORIGINAL ARTICLE

\title{
Transfer Time from the Intensive Care Unit and Patient Outcome: A Retrospective Analysis from a Tertiary Care Hospital in India
}

\author{
Sharmila Chatterjee ${ }^{1}$, Saswati Sinha², SK Todi ${ }^{3}$
}

\begin{abstract}
Background and aims: Patients' outcome after ICU transfer reflect hospital's post-ICU care status. This study assessed association of after-hour ICU transfer on patient outcome.

Subjects and methods: Single-centre, retrospective analysis of data between March2016 to April2017 at a tertiary-care hospital in India. Patient data were collected on all consecutive ICU admissions during study period. Patients were categorized according to ICU transfer time into daytime(08:00-19:59 hours) and after-hour(20:00-07:59 hours). Patients transferred to other ICUs/hospitals, died in ICU, or discharged home from ICU were excluded. Only first ICU admission was considered for outcome analysis. Primary outcome-hospital mortality; secondary outcomes-ICU re-admission and hospital length of stay (LOS). All analysis were adjusted for illness severity.

Results: Of 1857 patients admitted during study period, 1356 were eligible for study; $53.9 \%$ were males and $383(28 \%)$ patients transferred during after-hour. Mean age of two groups (daytime vs. after-hour $65.7 \pm 15.2$ vs. $66.3 \pm 16.2$ years) was similar ( $p=0.7$ ). Mean APACHE IV score was comparable between daytime vs. after-hour transfers $(45.6 \pm 20.4$ vs $46.8 \pm 22 ; p=0.05)$. Unadjusted hospital mortality rate of after-hour-transfers was significantly higher compared to daytime-transfers ( $7.1 \%$ vs. 4.1\%; $p=0.02)$. After adjustment with illness severity, after-hour-transfers were associated with significantly higher hospital mortality compared to daytime-transfers(aOR1.7, 95\%Cl 1.1,2.8; $p=0.04$ ). Median duration of hospital LOS and ICU re-admission though higher for after-hour-transfers, was not statistically significant in adjusted analysis (aOR ${ }^{\text {hospital- }}$

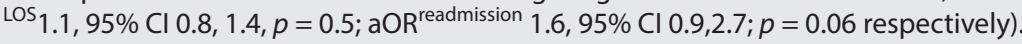

Conclusion: After-hour-transfers from ICU is associated with significantly higher hospital mortality. Hospital LOS and readmission rates are similar for daytime and after-hour -transfers.

Keywords: Intensive care, length of stay, mortality, readmission, transfer time Indian Journal of Critical Care Medicine (2019): 10.5005/jp-journals-10071-23132
\end{abstract}

\section{INTRODUCTION}

Intensive care unit (ICU) patients account for about $20 \%$ to $50 \%$ of in-hospital mortality rates ${ }^{1,2}$. Studies show approximately $10.8 \%$ ICU patients dying in general wards after being transferred from the ICU $\mathrm{IC}^{3,4}$. Safe and efficient transition of patients from ICU to the general ward often requires a proper transfer-out planning from the ICU ${ }^{5,7}$. Most deaths after ICU transfer have been attributed to higher disease severity (as evaluated with Acute Physiology and Chronic Health Evaluation (APACHE) scores), older age, organ failure and do-not-resuscitate orders ${ }^{3,4}$. However, recent studies also demonstrate an association between ICU transfer time to wards and hospital morality rates. After-hours transfer, defined as transfer from ICU at night and out-of-office hours have been reported to be associated with adverse hospital outcomes such as higher hospital mortality ${ }^{7,8}$, higher ICU readmission rate ${ }^{9,10}$, and prolonged hospital length of stay $(\operatorname{LOS})^{10}$. The excess mortality associated with afterhours transfer demonstrated in these studies also infer that the observed mortality is possibly due to premature transfer of patients or post-ICU suboptimal ward care in the after- hours ${ }^{7,10}$. Premature transfers are much more common at night ${ }^{11}$ and are generally the reflection of limited ICU bed capacity ${ }^{9,11-14}$, an increased demand for ICU beds or lack of nursing staff ${ }^{15}$.

Despite these results, some studies even failed to establish an association with after- hours transfer and hospital outcome. A Finnish multicenter study conducted in 18 ICUs did not find
Corresponding Author:Sharmila Chatterjee, e-mail:sharmilahotmail@ hotmail.com

How to cite this article: Chatterjee S, Sinha $S$ et al., Transfer Time from the Intensive Care Unit and Patient Outcome: A Retrospective Analysis from a Tertiary Care Hospital in India. Indian J of Crit Care Med 2019;23(3):115-121.

"out-of-office hour" transfer to be significantly associated with increased mortality after adjusting for illness severity, intensity of care, and presence of advanced directives (Odds ratio (OR) 1.1, $95 \%$ Confidence Interval (CI) $0.93-1.3)^{16}$. Another study also showed nighttime transfers to be associated with increased mortality in busy tertiary care hospitals ${ }^{17}$. Such discrepancies have been reported to be due to variations in nighttime definition, differences in patient population, study design and diverse local healthcare systems.

Patients' hospital outcome after ICU transfer depends upon post ICU care in the hospital and is often regarded as a quality indicator of the hospital. Furthermore, to date, there have been no studies from India that have investigated the effect of ICU transfer time on patient outcomes. We hypothesized that outcomes would be adverse for ICU patients transferred during after-hours compared to day-time transferred patients. A retrospective study was thus performed to assess the association of after-hour transfer from ICU to ward on subsequent hospital mortality, hospital LOS and ICU readmission.

(c) The Author(s). 2019 Open Access This article is distributed under the terms of the Creative Commons Attribution 4.0 International License (http://creativecommons. org/licenses/by/4.0/), which permits unrestricted use, distribution, and non-commercial reproduction in any medium, provided you give appropriate credit to the original author(s) and the source, provide a link to the Creative Commons license, and indicate if changes were made. The Creative Commons Public Domain Dedication waiver (http://creativecommons.org/publicdomain/zero/1.0/) applies to the data made available in this article, unless otherwise stated. 
Transfer Time from the Intensive Care Unit and Patient Outcome

\section{Methods}

This retrospective cohort study was conducted in a 23 bedded ICU of a tertiary care hospital in India over a 14 month period between March, 2016 to April, 2017. The study was approved by the Institutional Review Board and informed consent was waived because the study was observational in nature, and use of de-identified data from a pre-existing ICU database.

\section{Study Population}

The study included all adult patients ( $\geq 18$ years age) consecutively admitted to the ICU with $\geq 24$ hours of ICU stay during study period, and discharged alive from ICU to wards. Patients who died in the ICU, transferred to other hospitals or nursing homes, discharged home from ICU or discharged against medical advice were excluded. Patients with missing data were also excluded. Only the first admission was considered for patients with multiple ICU admissions during a single hospitalization.

\section{Definition of Variables}

For the purpose of this study, we defined "after-hour" transfer as transfer from ICU occurring between 08:00 pm and 07:59 am, and "daytime" transfer as transfer from ICU between 08:00 am and 07:59 pm. ICU readmission was defined as patients' getting back to ICU from wards within the current hospitalization irrespective of the time between their ICU discharge and re-admission to ICU. ICU admission source was categorized into those arriving from the emergency, ward, other hospitals, operation theaters, and high dependency units (HDU). Admission diagnosis was used to classify the body system involved. Presence of any cardiovascular disease, chronic obstructive airway disease, diabetes mellitus, neurological diseases and chronic kidney diseases were the major co-morbidities considered in our study. The APACHE IV score was used to determine severity of illness on admission. The APACHE IV determines the severity of illness by including a number of physiological, biochemical and demographic (e.g. age, blood pressure and chronic health status) variables to calculate a numerical score to assess the predicted risk of death ${ }^{18}$. Hospital LOS was defined as the total number of days that a patient stayed in hospital, including ICU stay, from the time of admission. ICU support status was determined by whether patients required invasive mechanical ventilation (IMV), non-invasive mechanical ventilation (NIMV), ionotrope/vasopressor support or blood transfusion during their ICU stay.

Other variable information collected included patient demographics and ICU discharge status (patients discharged alive or dead, whether discharged home, or to the ward, other ICUs or high dependency units (HDU), or discharged against medical advice) and hospital discharge status as discharged alive or dead.

\section{Data Source and Data Quality}

Inpatient information was extracted from the ICU database. The database regularly updated by two trained data entry operators, contain information on 220 variables of ICU patients. The data entry operators scrutinize in-patient records and investigation files daily to collect information on all relevant variables. Two full-time ICU consultants supervise the collected data daily. Any data discrepancy in the database is cross-checked with patient records for errors during entry. For this study, all patient personal identifiers were removed from data files to maintain patient confidentiality and all patients in the database were provided with a unique ID number.
Quality of data was assessed by screening 50 randomly selected records every month. Any difference was resolved by consensus. All data were collected on a pre formed structured Excel data sheet.

\section{Statistical Analysis}

Time of ICU discharge (after-hours vs. daytime) was the primary exposure. The primary outcomes of our study were crude and risk- adjusted in-hospital mortality and secondary outcomes were hospital LOS and ICU readmissions. Descriptive data has been expressed as mean (SD), median (interquartile range), or percentages. Student's $t$ test was used to describe normally distributed data while Wilcoxon's rank sum test was used for analyzing nonparametric data. Chi-square test was used to analyze categorical data. Bivariate and multivariable logistic regression models were used to estimate crude and risk adjusted estimates for "after-hours" transfer risk, with adjustment for APACHE IV and other co-variates. Results were reported as odds ratios and $95 \%$ confidence interval. Two-tailed tests were used with a significance level at $a=0.05$. All statistical analysis was performed using the PC-SAS program (V9.2, SAS Institute, Cary, NC, USA).

\section{Results}

There were a total of 1857 patients admitted during study period. After excluding patients who were discharged home from ICU directly $(n=73)$, or discharged against medical advice (DAMA) ( $n=108)$, and patients who died in the ICU $(n=108)$, survival till ICU transfer to ward occurred in $77.5 \%(n=1439)$ of admitted patients. Another 83 (4.4\%) were excluded due to missing data and the final study population consisted of 1356 patients. Of these, 383 (28.2\%) transfers occurred during after-hours, from 08:00 pm to 07:59 am, and 973 (71.8\%) were daytime transfers (from 08:00 am to 07:59 pm) (Figure 1). 69 (3.7\%) patients withdrew or withheld life sustaining treatment in the ICU. While majority (92.8\%) of them either expired in ICU or were discharged against medical advice , 5 patients were transferred to the ward out of which 1 patient was eventually discharged from hospital.

The mean age of patients transferred during after-hours did not significantly differ from those transferred during daytime $(66.3 \pm 16.2$ vs. $65.7 \pm 15.2$ years, $p=0.7)$. The sex distribution of patients was also similar in the two transfer periods (male: $55.4 \%$ vs. $53.3 \% ; p=0.5$ ). Significantly higher number of patients transferred during after-hours were admitted from the HDU (3.7\% vs. $1.9 \% ; p=0.05$ ) compared to daytime transferred patients. Daytime transferred patients were more commonly admitted from the operating theaters $(13.8 \%$ vs. $8.1 \% ; p=0.004)$. There was no significant difference in co-morbidities between after-hour and daytime transfers $(77.6 \%$ vs. $77 \% ; p=0.6)$. Mean APACHE IV scores and predicted mortality rates were significantly higher for afterhour transfer patients compared to day time transfer patients respectively ( $46.8 \pm 22$ vs. $45.6 \pm 20.4, p=0.05 ; 9.7 \%$ vs.7.8\%, $p=0.009$ ). After- hour transfer patients were sicker patients and more likely to have received mechanical ventilation and ionotrope support $(18.5 \%$ vs. $13.5 \%, p=0.03 ; 19.3 \%$ vs. $11.6 \%$, $p=0.0003$ ) during their ICU stay. Significantly higher number of patients with diagnosis of sepsis were transferred during afterhours $(6.8 \%$ vs $2.9 \% ; p=0.0009)$. The characteristics of all patients transferred during daytime and after-hours are described in Table 1. Hospital mortality was significantly higher among after-hour transferred patients compared to daytime transferred patients 
Transfer Time from the Intensive Care Unit and Patient Outcome

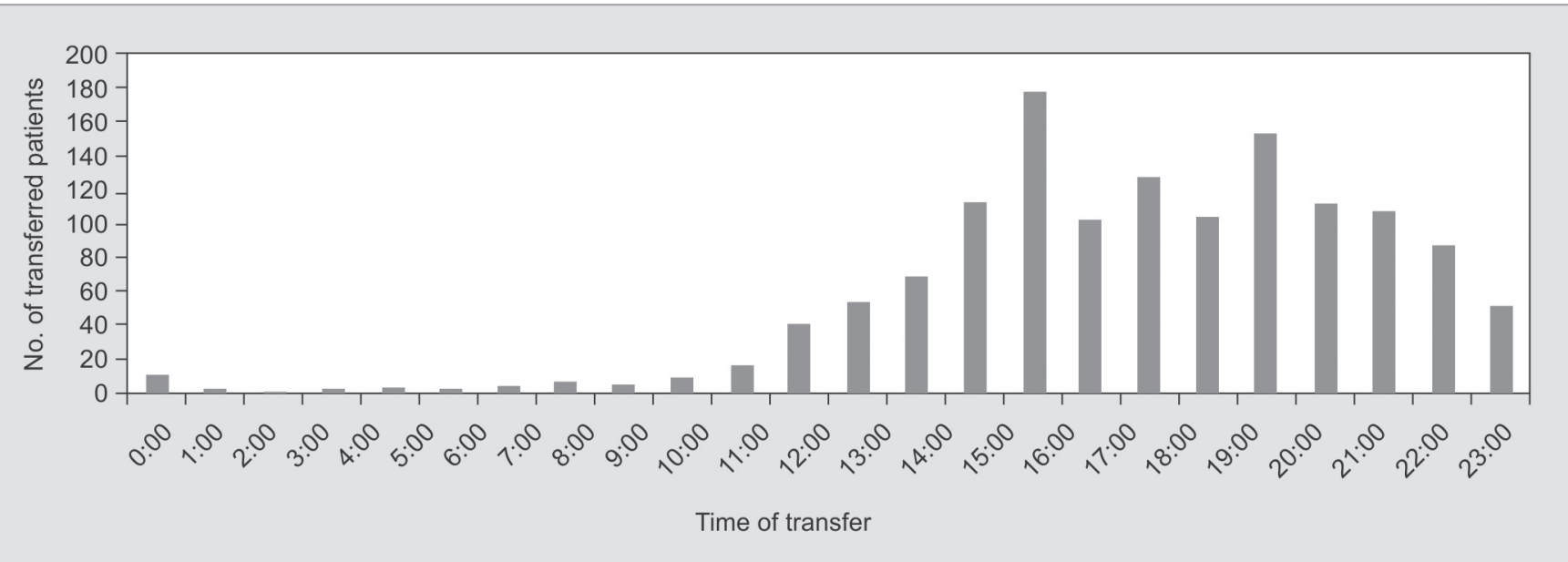

Fig. 1: Showing the number of patients transferred out of ICU by hour

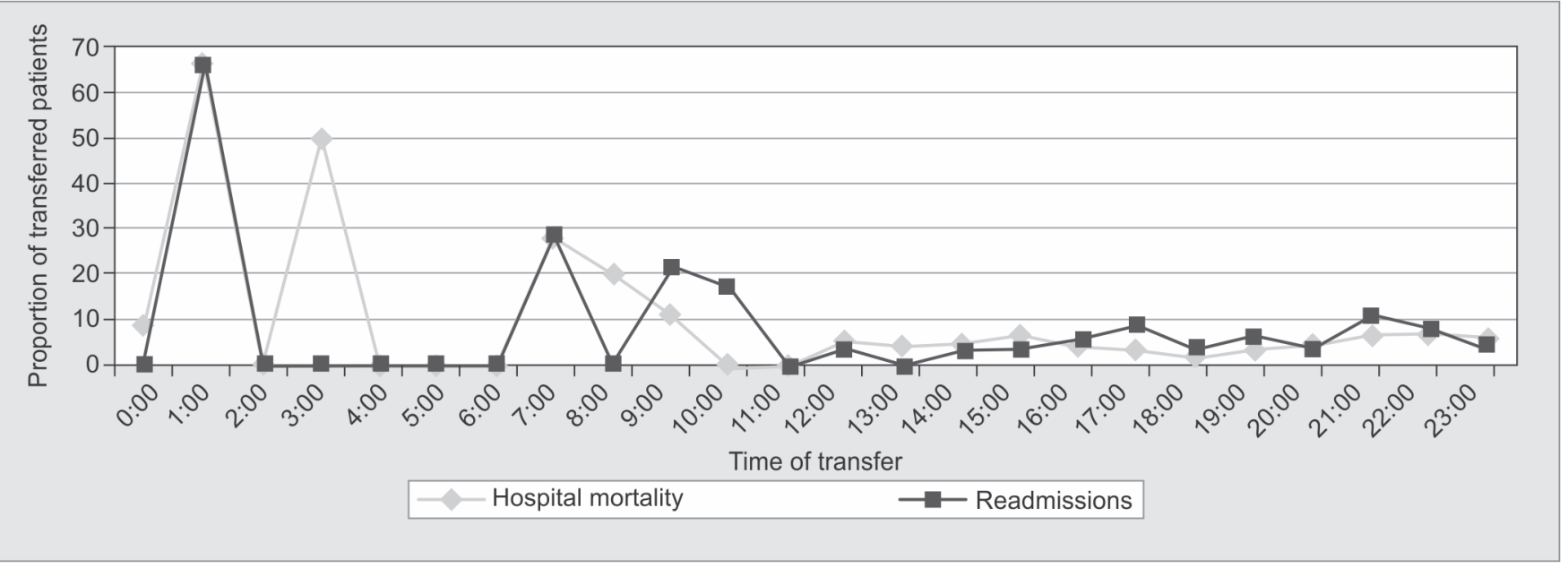

Fig. 2: Showing hospital mortality and readmission rates of transferred patients by hour

(7.1\% vs.4.1\%, $p=0.02$ ). ICU readmissions and the median LOS in hospital also differed significantly among the two transfer groups (Table 2, Figure 2).

\section{After hour transfer and hospital mortality, ICU readmission and hospital LOS}

On bi-variate analysis, factors that were seen to be associated with increased risk of hospital mortality were higher age (OR 1.8, 95\% Cl 1.1-3.0, $p=0.02$ ), presence of co-morbidities (OR 2.5, 95\% Cl 1.1-5.6, $p=0.02)$, higher APACHE IV score at admission (OR 1.2, 95\% Cl 1.1-1.6, $p<0.0001)$, receipt of ICU support (OR 1.8, 95\% Cl 1.1-3.0, $p=0.02$ ) and after-hour transfer (OR 1.8, 95\% Cl 1.1-3.0, $p=0.02$ ) (Table 3). However, adjusted analysis in the multivariable logistic regression model showed that after-hour transfer remained the single most significant independent factor associated with hospital mortality (aOR 1.7, $95 \% \mathrm{Cl} 1.1-2.8, p=0.04$ ) (Table 4). After-hour transfer was also associated with higher odds of readmission and hospital LOS, but this was not statistically significant in the adjusted model (aOR ${ }^{\text {readmission }} 1.6,95 \% \mathrm{Cl}$ 0.99-2.6, $p=0.06$; aOR ${ }^{\text {hospital LOS }} 1.195 \%$ $\mathrm{Cl} 0.8-1.4, p=0.5$ ).

Of the 383 patients transferred out of the ICU at after-hours, 43 (11.2\%) had an adverse outcome - death or ICU readmission. These patients significantly differed from those who were eventually discharged alive in terms of their mean age, APACHE IV scores, co-morbidities, ICU support status and predicted mortality rates (PMR). Differences between the after-hour discharged patients with and without adverse outcome are listed in Table 5.

\section{Discussion}

Our study demonstrated increased mortality (crude and riskadjusted) associated with after-hour transfer from ICU compared to daytime transferred patients. These findings are in agreement with findings from Goldfrad and Rowan in UK ${ }^{11}$ who first demonstrated that patients transferred during after-hours had a significantly higher crude as well as case mix adjusted mortality compared to daytime transfers (crude OR 1.46, 95\% CI 1.18-1.80 and case-mix adjusted OR $1.33,95 \% \mathrm{Cl} 1.06-1.65)$. They postulated that the increase in mortality among after-hour transfer patients was a reflection of rising demand on ICU beds leading to premature ICU discharges ${ }^{11}$. The adverse impact of after-hour discharges were further documented by several studies from UK ${ }^{19}$, USA ${ }^{10}$, Australia ${ }^{7-9,14,19}$ and Canada ${ }^{15,20}$. After-hour ICU discharge, not considered optimal care or standard practice, is often due to 
Transfer Time from the Intensive Care Unit and Patient Outcome

\begin{tabular}{|c|c|c|c|}
\hline Characteristics & Daytime $(n=973)$ & After-hour $(n=383)$ & $P$ \\
\hline Age, mean $\pm S D$ & $65.7 \pm 15.2$ & $66.3 \pm 16.2$ & 0.7 \\
\hline Gender, male, n (\%) & $519(53.3)$ & $212(55.4)$ & 0.5 \\
\hline BMla, mean \pm SD & $22.2 \pm 2.7$ & $22.2 \pm 2.6$ & 0.2 \\
\hline \multicolumn{4}{|l|}{ Source of admission n(\%) } \\
\hline Emergency & $628(64.4)$ & $265(69.2)$ & 0.1 \\
\hline Ward & $142(14.6)$ & $51(13.3)$ & 0.5 \\
\hline OTb & $134(13.8)$ & $31(8.1)$ & $0.004^{*}$ \\
\hline Other hospitals & $9(0.9)$ & $4(1.04)$ & 0.8 \\
\hline HDUC & $18(1.9)$ & $14(3.7)$ & $0.05^{*}$ \\
\hline Other ICU & $42(4.3)$ & $18(4.7)$ & 0.8 \\
\hline \multicolumn{4}{|l|}{ Admission diagnosis n(\%) } \\
\hline Cardiovascular & $94(9.7)$ & $30(7.8)$ & 0.3 \\
\hline Respiratory & $208(21.4)$ & $80(20.9)$ & 0.8 \\
\hline Gastro-intestinal \& hepatobiliary & $194(19.9)$ & $66(17.2)$ & 0.3 \\
\hline Genito urinary & $189(19.4)$ & $69(18)$ & 0.6 \\
\hline Neurological & $47(3.5)$ & $24(1.8)$ & 0.3 \\
\hline Infectious diseases & $25(2.6)$ & $16(4.2)$ & 0.2 \\
\hline Sepsis (all causes) & $28(2.9)$ & $26(6.8)$ & $0.0009^{*}$ \\
\hline Metabolic & $64(6.6)$ & $28(7.3)$ & 0.6 \\
\hline Trauma & $12(1.2)$ & $8(2.1)$ & 0.6 \\
\hline Dermatology & $3(0.3)$ & 0 & 0.3 \\
\hline Hematology & $19(1.9)$ & $6(1.6)$ & 0.2 \\
\hline Musculoskeletal & $65(6.7)$ & $18(4.7)$ & 0.2 \\
\hline \multicolumn{4}{|l|}{ Operative status n(\%) } \\
\hline Medical & $803(82.5)$ & $337(88)$ & $0.05^{*}$ \\
\hline Comorbidities n(\%) & $759(78)$ & $297(77.6)$ & 0.6 \\
\hline Cardiovascular diseases & $629(64.6)$ & $247(64.5)$ & 0.9 \\
\hline Diabetes Mellitus & $404(41.5)$ & $158(41.3)$ & 0.9 \\
\hline Chronic obstructive airway diseases & $152(15.6)$ & $53(13.8)$ & 0.4 \\
\hline Chronic kidney diseases & $102(10.5)$ & $50(13)$ & 0.2 \\
\hline Neurological Diseases & $89(9.2)$ & $45(11.8)$ & 0.1 \\
\hline APACHE IV, mean \pm SD & $45.6 \pm 20.4$ & $46.8 \pm 22$ & $0.05^{*}$ \\
\hline ICU support, $\mathrm{n}(\%)$ & $344(35.4)$ & $169(44.1)$ & $0.003^{*}$ \\
\hline IMVd & $134(13.8)$ & $71(18.5)$ & $0.03^{*}$ \\
\hline NIMVe & $102(10.5)$ & $51(13.3)$ & 0.1 \\
\hline Noradrenalin & $113(11.6)$ & $74(19.3)$ & $0.0003^{*}$ \\
\hline Vasopressin & $3(0.3)$ & $4(1.04)$ & 0.1 \\
\hline Blood transfusion & $171(17.6)$ & $66(17.2)$ & 0.9 \\
\hline Predicted mortality rate & 7.8 & 9.7 & $0.009^{*}$ \\
\hline
\end{tabular}

a. BMI: Body mass index

b. OT: Operation theatre

c. HDU: High dependency unit

d. IMV Invasive mechanical ventilation

e. Non invasive mechanical ventilation

Table 2: Outcome differences between after-hour and daytime transfers

\begin{tabular}{llll}
\hline Outcome variables & Daytime $(n=973)$ & After-hour $(n=383)$ & $P$ \\
\hline Hospital mortality, $\mathrm{n}(\%)$ & $40(4.1)$ & $27(7.1)$ & $0.02^{*}$ \\
ICU readmission, $\mathrm{n}(\%)$ & $48(4.9)$ & $29(7.6)$ & $0.05^{*}$ \\
$\begin{array}{l}\text { Hospital length of stay, days } \\
\text { median (IQR) }\end{array}$ & $8(6-13)$ & $9(6-14)$ & $<0.001^{*}$ \\
\hline
\end{tabular}


Transfer Time from the Intensive Care Unit and Patient Outcome

Table 3: Odds ratios (OR) and corresponding $95 \%$ confidence intervals (CI) for clinical variables associated with hospital mortality after ICU transfer

\begin{tabular}{llll}
\hline Clinical factors & $O R$ & $95 \% \mathrm{Cl}$ & $P$ \\
\hline Age & 1.02 & $1.01-1.04$ & $0.01^{*}$ \\
Sex (ref = male) & 0.6 & $0.4-1.01$ & 0.06 \\
Comorbidities & 2.5 & $1.1-5.6$ & $0.02^{*}$ \\
APACHE IV & 1.2 & $1.1-1.6$ & $0.0001^{*}$ \\
ICU support & 1.8 & $1.1-3$ & $0.02^{*}$ \\
Source of admission & 1.02 & $0.9-1.2$ & 0.8 \\
Emergency & 1.1 & $0.8-1.2$ & 0.2 \\
Ward & 1.1 & $0.6-2.2$ & 0.7 \\
HDU & 8.7 & $3.8-19.6$ & $<0.0001^{*}$ \\
OT & 0.8 & $0.4-1.7$ & 0.5 \\
Other hospitals & 4.1 & $0.9-10.1$ & 0.7 \\
Admission diagnosis & & & 0.7 \\
Cardiovascular & 1.2 & $0.5-2.6$ & 0.7 \\
Respiratory & 1.1 & $0.6-2$ & 0.4 \\
Gastrointestinal \& hepatobiliary & 1.3 & $0.7-2.3$ & 0.2 \\
Genito urinary & 1.4 & $0.8-2.5$ & 0.2 \\
Neurological & 0.3 & $0.04-2.0$ & 0.9 \\
Infectious diseases & 1.01 & $0.3-2$ & 0.4 \\
Sepsis (all causes) & 1.6 & $0.6-4.6$ & 0.8 \\
Metabolic & 1.1 & $0.4-2.8$ & 0.9 \\
Trauma & 0.6 & $0.08-2.3$ & $0.02^{*}$ \\
Transfer time (ref = daytime) & 1.8 & $1.1-3.0$ & \\
\hline
\end{tabular}

Table 4: (Adjusted) Multivariable logistic regression analysis showing the independent association of transfer time with hospital outcome

\begin{tabular}{lllll}
\hline Predictor variable - Time of transfer & Outcome variable & OR & $95 \% \mathrm{Cl}$ & $P$ \\
\hline Daytime & Hospital mortality & Reference & & $0.04^{*}$ \\
After-hour & & 1.7 & $1.1-2.8$ & 0.06 \\
Daytime & ICU readmission & Reference & & \\
After-hour & & 1.6 & $0.99-2.7$ & 0.5 \\
Daytime & Hospital LOS & Reference & & $0.8-1.4$ \\
After-hour & & 1.1 & & \\
\hline
\end{tabular}

Table 5: Differences between after-hour transfers with and without adverse outcome

\begin{tabular}{llll}
\hline Age, co-morbidities and severity of illness & $\begin{array}{l}\text { Alive at hospital discharge without ICU } \\
\text { readmission }(n=340)\end{array}$ & $\begin{array}{l}\text { Dead at hospital discharge or readmission } \\
\text { to the ICU }(n=43)\end{array}$ & $P$ \\
\hline Age, mean \pm SD & $65.4 \pm 16.6$ & $71.6 \pm 11.9$ & $0.01^{*}$ \\
Comorbidities, $n(\%)$ & $255(75)$ & $42(97.7)$ & $0.0008^{*}$ \\
APACHE IV & $48.8 \pm 23.4$ & $54.7 \pm 20.6$ & 0.3 \\
ICU support & $141(41.5)$ & $28(65.1)$ & $0.003^{*}$ \\
Predicted mortality rate & 8.6 & 13 & $<0.0001^{*}$ \\
\hline
\end{tabular}

increased pressure on ICU beds to accommodate a new patient, or organizational aspects such as unavailability of a bed in the ward until late hours. Both these factors are likely to have played a role in our study as the average ICU occupancy rates are high causing premature discharges, and delays in transfer occur when designated bed in the ward to which the patient is to be transferred is not vacant. Moreover less surveillance, lower nurse-to-patient ratios and decreased staff availability in the wards during after-hours could also attribute to increased mortality for patients transferred from $\mathrm{ICU}^{14}$. Transfers in the middle of the night may cause both physical and psychological trauma to patients ${ }^{11}$.
Our study findings however differ from a Finnish study from 18 university and central hospital ICUs which failed to demonstrate an association between after-hour transfer and increased mortality ${ }^{16}$. The difference could be due to differing after-hour definitions and methods for assessing illness severity. The Finnish study adjusted for illness severity using the SAPS II-scores and defined after-hour as 16:00 hours to $08: 00$ hours. In contrast, our study used APACHE IV score for assessing illness severity and defined after-hour as 20:00 hours to 08:00 hours. Two other studies also did not find any relation between ICU discharge time and mortality. While the first study ${ }^{10}$ stated adequate availability of ICU beds in their setting 
making premature transfers unlikely and also optimum staffing round the clock, the latter ${ }^{21}$ had a different methodological design.

The proportion of night time discharges in our study (28.2\%) was higher compared to the mean of $15.3 \%$ (range:3.6\% to $34.7 \%$ ) in a recently reported meta analysis ${ }^{22}$. There was no significant difference in the demographic characteristics between patients transferred during daytime and those transferred during afterhours and they were also similar in terms of baseline co-morbidities. However a higher proportion of patients admitted to ICU after surgery from the operation theaters were transferred to ward during daytime. This likely represents a group of elective admissions who were probably kept for post operative monitoring and their transfer planning was therefore relatively straightforward. As opposed to this, patients transferred during after-hours had higher APACHE IV scores with higher predicted mortality, and they were more likely to have sepsis and organ support requirement in terms of mechanical ventilation and/or vasopressors. This constitutes a sicker population of patients and as shown in earlier studies ${ }^{8,13}$ might explain increased risk of mortality associated with transfers during after-hours.

Crude bi-variate analysis showed association of older age, presence of co-morbidities, APACHE IV score and organ support (ICU support) with increased hospital mortality. However, after adjustment for these variables, after-hour transfer remained the single independent predictor of hospital mortality. This has also been convincingly demonstrated by Yang et al. ${ }^{22}$ in their meta analysis of fourteen studies which showed night time discharge to significantly impact mortality even after adjustment for disease severity at ICU admission and discharge. Unadjusted analysis in our study also showed a higher ICU readmission rate as well as a longer hospital LOS for patients transferred during after-hours compared to daytime transferred patients. However both these secondary outcomes did not achieve statistical significance in the adjusted analysis.

Several factors that contribute to a patients outcome following ICU discharge include co-morbidities, age and APACHE IV score not only at time of admission but also at time of transfer, as well as continuing organ support requirement after ICU discharge. The staffing of wards both in terms of manpower and ability to provide continuity of care is of paramount importance. It has been shown that presence of organ dysfunction as well as requirement of higher levels of support following ICU discharge could contribute to higher mortality ${ }^{22}$. Intensivists should thus recognize this high-risk group and exercise caution while transferring such patients from the ICU in after-hours when continuity of care might be compromised. Placing limitations on care and resuscitation measures may also impact outcome of such sicker patients after ICU discharge. Finally in the Indian scenario, financial constraints influencing decisions to transfer patients prematurely from the ICU when a patient would ideally have benefited from 24-48 hours of additional ICU care, is a common phenomenon and can impact patient outcome.

Our study has limitations. The study was observational in nature with retrospective analysis thereby limiting us to information that was already collected in the database. Severity of illness at ICU discharge is an important factor influencing post ICU outcome. Our study did not have this data. This was a single-centered study. Our institute also has unique organizational characteristics such as limited ICU and HDU beds, and limited skilled manpower in after-hours in the ward - both medical and nursing especially for high-risk patients. The scenario from our ICU thus, might not be generalizable to other hospitals where rapid response teams, ICU outreach services or medical emergency teams are available who can provide extension of ICU care to ward patients round the clock. Also limitation of medical treatment is a major subgroup of patients which has been analyzed in almost all prior studies; however we had few such patients and excluded them from our analysis. Finally in an observational study finding an association cannot be interpreted as causation. However, this is the first study from India to study association of ICU transfer time and outcome. The findings from this study may help physicians take decisions on after-hour transfer of patients.

In conclusion, this study has identified and also supports an association between after-hour transfer and mortality of ICU survivors. Further research will likely identify precise factors, linking mortality associated with ICU transfer time and the organizational processes that could be put in place to reduce mortality rates.

\section{Acknowledgements}

We are grateful to all ICU consultants, doctors and nursing staff for their help during data collection process, and the data entry operators, Ms.Shibani Kayal and Ms. Chandrani Das for collection of data and developing the electronic database for this research.

\section{References}

1. Knaus WA, Wagner DP, Draper EA, Zimmerman JE, Bergner M, Bastos PG, et al. The APACHE III prognostic system. Risk prediction of hospital mortality for critically ill hospitalized adults. Chest 1991;100(6):161936.

2. Beck DH, Taylor BL, Millar B, Smith GB. Prediction of outcome from intensive care: a prospective cohort study comparing Acute Physiology and Chronic Health Evaluation II and III prognostic systems in a United Kingdom intensive care unit. Crit Care Med 1997;25(1):9-15.

3. Azoulay E, Adrie C, De Lassence A, Pochard F, Moreau D, Thiery G, et al. Determinants of postintensive care unit mortality: a prospective multicenter study. Crit Care Med 2003; 31(2): 428-32.

4. Moreno R, Miranda DR, Matos R, Fevereiro T. Mortality after discharge from intensive care: the impact of organ system failure and nursing workload use at discharge. Intensive Care Med 2001;27 (6):999-1004.

5. Durbin CG, Kopel RF. A case-control study of patients readmitted to the intensive care unit. Crit Care Med 1993;21:1547-53.

6. Rosenberg AL, Watts CW. Patients readmitted to ICUs. A systematic review of risk factors and outcomes. Chest 2000;118:492-502.

7. Beck DH, McQuillan P, Smith GR. Waiting for the break of dawn? The effects of discharge time, discharge TISS scores and discharge facility on hospital mortality after intensive care. Intensive Care Med 2002;28:1287-93.

8. Singh MY, Nayyar V, Clark PT, Kim C. Does after-hours discharge of ICU patients influence outcome? Crit Care Resusc 2010;12(3):156-61.

9. Pilcher DV, Duke GJ, George C, Bailey MJ, Hart G. After-hours discharge from intensive care increases the risk of readmission and death. Anaesth Intensive Care 2007;35(4):477-85.

10. Hanane T, Keegan MT, Seferian EG, Gajic O, Afessa B. The association between nighttime transfer from the intensive care unit and patient outcome. Crit Care Med 2008;36(8):2232-7.

11. Goldfrad C, Rowan K. Consequences of discharges from intensive care at night. Lancet 2000; 355:1138-42.

12. Obel N, Schierbeck J, Pedersen L, Storgaard M, Pedersen C, Sorensen $\mathrm{HT}$, et al. Mortality after discharge from the intensive care unit during the early weekend period: a population-based cohort study in Denmark. Acta Anaesthesiol Scand 2007;51(9):1225-30.

13. Laupland KB, Shahpori R, Kirkpatrick AW, Stelfox HT. Hospital mortality among adults admitted to and discharged from intensive care on weekends and evenings. J Crit Care 2008; 23(3):317-24. 
14. Duke GJ, Green JV, Briedis JH. Night-shift discharge from intensive care unit increases the mortality-risk of ICU survivors. Anaesth Intensive Care 2004;32(5):697-701.

15. Priestap FA, Martin CM. Impact of intensive care unit discharge time on patient outcome. Crit Care Med 2006;34(12):2946-51.

16. Uusaro A, Kari A, Ruokonen E: The effects of ICU admission and discharge times on mortality in Finland. Intensive Care Med 2003;2144-48.

17. Bristow PJ. Discharges out of hours are associated with increased mortality. Anaesth Intensive Care 2003;31:222.

18. Dahhan T, Jamil M, Al-Tarifi A, Abouchala N, Kherallah M. Validation of the APACHE IV scoring system in patients with severe sepsis and comparison with the APACHE II system. Crit Care 2009;13(Suppl 1):P511.
19. Tobin $A E$, Santamaria JD. After-hours discharges from intensive care are associated with increased mortality. Med J Aust 2006;184(7):334-7.

20. Azevedo LC, de Souza IA, Zygun DA, Stelfox HT, Bagshaw SM. Association between nighttime discharge from the intensive care unit and hospital mortality: a multi-center retrospective cohort study. BMC Health Serv Res 2015;15:378.

21. Santamaria JD, Duke GJ, Pilcher DV, Cooper DJ, Moran J, Bellomo R; Discharge and readmission evaluation (DARE) study.The timing of discharge from the intensive care unit and subsequent mortality. A prospective, multicenter study. Am J Respir Crit Care Med 2015; 191(9): 1033-9.

22. Yang S, Wang Z, Liu Z, Wang J, Ma L. Association between time of discharge from ICU and hospital mortality: a systematic review and meta-analysis. Crit Care 2016;20(1):390. 